

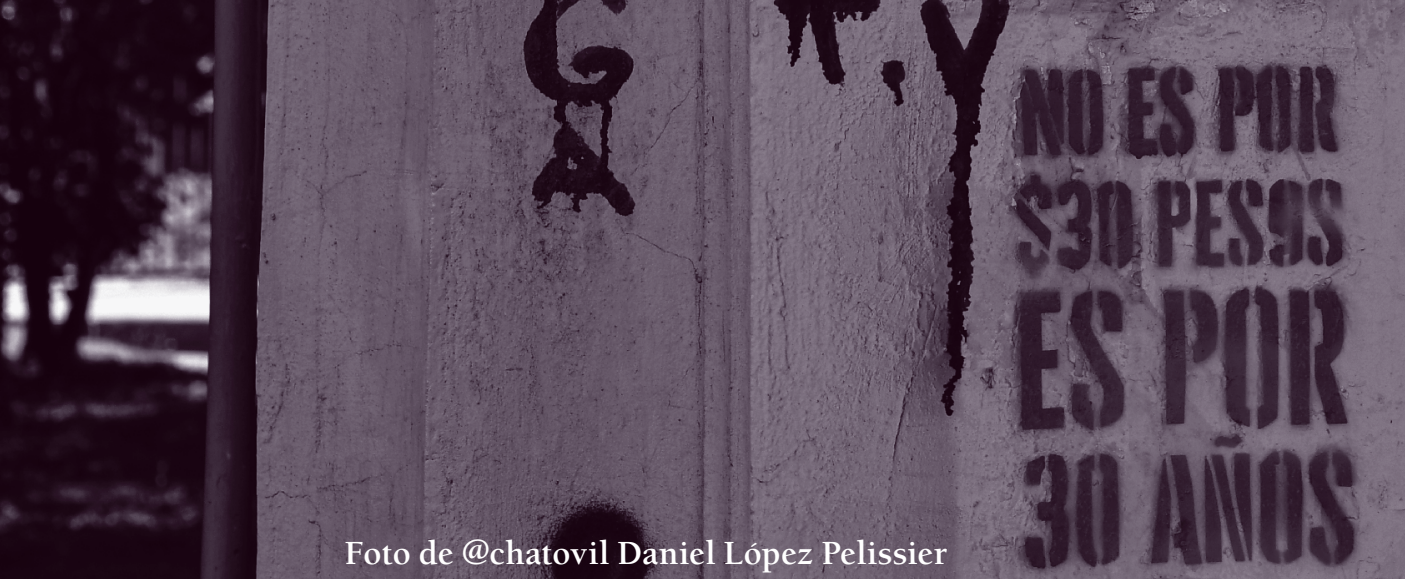

\section{Desigualdades urbanas: costos y tiempos de viaje en el Área Metropolitana de Santiago}

Palabras clave: costos de movilidad, inequidad territorial, tiempos de viaje, segregación socioespacial, Santiago de Chile

\section{Resumen}

La expansión urbana ha ampliado las distancias y tiempos de viaje de las personas que viven y trabajan en las ciudades. En el Área Metropolitana de Santiago de Chile sus habitantes menos favorecidos enfrentan profundos desafíos, pues tanto sus ingresos como los territorios donde residen revelan una menguada disponibilidad de capitales de movilidad. La presente investigación busca calcular y entender los distintos costos de la movilidad expresados en tiempo y en dinero, a partir de los viajes al trabajo como expresión de las desigualdades urbanas existentes en la ciudad. Se utilizan para esto las Encuestas de Caracterización Socio Económica (CASEN) del Ministerio de Desarrollo Social de 2015 y 2017, que incorporan preguntas sobre la movilidad de las personas. Desde un enfoque cuantitativo, se definen los costos monetarios mensuales de la movilidad de los hogares determinando su porcentaje en el ingreso monetario del hogar y se realiza un análisis complementario considerando distintas variables socioeconómicas. Los resultados evidencian profundas desigualdades debidas al mayor peso del costo del transporte público en los ingresos de los más vulnerables, hecho que se condice con mayores tiempos de viaje debido a la lejanía entre su residencia y los centros laborales, mayor desempleo y menor motorización privada. 


\section{*.*vis revistainvi}

\section{Introducción}

Producto de la expansión urbana, las distancias y tiempos de viaje han ido aumentando en las áreas metropolitanas, profundizando las condiciones de segregación socio espacial y de creciente desigualdad en términos de conectividad de los territorios. Tanto la localización residencial como las competencias de movilidad, así como las necesidades y motivos para desplazarse, son variables determinantes de la movilidad cotidiana de las personas.

El paradigma de la movilidad reconoce el papel que juegan las distintas formas en que la movilidad se manifiesta en las ciudades, en términos de inclusión social y equidad. La movilidad considerada como variable social expresa las modalidades a través de las cuales las personas se mueven en las distintas esferas de su vida cotidiana. Desde este enfoque, el nivel de accesibilidad corresponde a la capacidad de movilidad, la cual queda determinada tanto por las condiciones socioeconómicas de los habitantes como por las condiciones de conectividad que ofrecen los distintos sectores de la ciudad.

En el Área Metropolitana de Santiago, la localización de los servicios y fuentes laborales, concentrados en el centro, la zona oriente y en algunas periferias industriales, no coincide con la localización residencial de la mayoría de los habitantes (Jirón y Mansilla, 2014). En ese contexto, la ecuación entre costos de transporte y localización residencial no siempre es optimizada -como esperan los tradicionales modelos neoclásicos (Alonso, 1964; Wingo, 1961)-, sino que, por el contrario, genera una pérdida social expresada en pérdida de accesibilidad. Es en los hogares más desfavorecidos donde se expresa con mayor notoriedad esta pérdida de capacidad de movilidad y de accesibilidad, en especial, respecto de las zonas donde se concentran las oportunidades de la ciudad.

Según nuestra hipótesis, los mayores costos de viaje (en tiempo y en costo monetario) se asocian a las condiciones socioeconómicas del territorio (localización, precios de suelo, accesibilidad) altamente segregado y por eso se concentran en la población y en las zonas de más bajos ingresos de las comunas del Gran Santiago, reproduciendo la segregación y la desigualdad.

Para demostrar esto, se ha recurrido al procedimiento de calcular los costos de viaje de los hogares de acuerdo a sus características, tamaño y nivel de ingreso. Se ha ocupado la Encuesta de Caracterización Socio Económica (CASEN) del Ministerio de Desarrollo Social de Chile, en sus versiones 2015 y 2017 que incorporan datos sobre los viajes de las personas al trabajo, representativos para cada decil de ingreso. Esta información ha sido expandida para viajes del grupo familiar del hogar, estimando la composición del grupo y sus desplazamientos. A partir del cálculo del total de viajes de cada familia representativa de cada decil de ingreso, se determina su peso en el ingreso monetario del hogar, que considera todos los ingresos, provenientes de sueldos y salarios, monetarios y en especie, además de subsidios; asimismo, se desarrolla un análisis complementario considerando variables de localización comunal, que se vinculan tanto con el ingreso como con las condiciones de empleo, el tiempo de viaje y la motorización. De esta manera, se demuestra que existe 


\section{*.*vis revistainvi}

una correlación inversa entre el nivel de ingreso y el peso del costo de transporte según deciles de ingreso. Del mismo modo, se muestra también que entre el nivel de ingreso y el tiempo de viaje existe una similar correlación inversa.

El presente trabajo se divide en seis partes, incluyendo la introducción. En la segunda, se presenta la problemática y el estado del arte, donde se realiza una discusión teórica sobre distintos conceptos del capital de la movilidad, la accesibilidad y las desigualdades urbanas. En la tercera se plantea la metodología y fuentes de información utilizadas. En la cuarta se muestran los principales resultados expresados en tablas, mapas y gráficos y su correspondiente análisis. En la quinta se desarrolla la discusión de los resultados contrastándose con otros trabajos previos. Finalmente, en la sexta, se plantean las principales conclusiones.

\section{Problemática y estado del arte}

En las últimas décadas, la movilidad ha sido considerada como objeto de estudio y como prisma de análisis de las sociedades modernas (Ascher, 1995; Bauman, 2000). Estas líneas de investigación tuvieron por objetivo abrir el campo de estudio hacia la incidencia de la movilidad en las diferentes dimensiones tanto espaciales como socioeconómicas, analizando el capital de movilidad de las personas dentro de nuestras sociedades, lo que Kaufmann (2008) identifica con el concepto de motilidad. Siguiendo a Kaufmann, la posibilidad de moverse en nuestras sociedades urbanas, cada vez más complejas y diversas, condiciona el acceso a los recursos y oportunidades que nos ofrece la ciudad. Nos movemos en una sociedad de redes en la cual, no solo las experiencias de movilidad, sino también las experiencias sociales y las oportunidades que genera la movilidad, son cada vez más híbridas (Sheller y Urry, 2006). Las movilidades contemporáneas aparecen así como una consecuencia y a su vez como factores de individualización y diversificación de las prácticas sociales. En este nuevo paradigma, la movilidad se vuelve un hecho social en sí.

Bajo ese prisma de análisis, el debate sobre la importancia de las prácticas de movilidad en la inclusión social se ha ido posicionando a nivel teórico (Garretón, 2011; Jirón et al., 2010; Lucas, 2012; Preston, 2009; Sheller, 2018; Wenglenski, 2010). Se desarrolló así un campo teórico importante centrado en el estudio del rol que tiene la movilidad cotidiana para la inclusión social, reconociendo el transporte como componente clave para entregar a los sectores más desfavorecidos mayor accesibilidad a las áreas de oportunidades económicas, sociales y culturales que ofrece la ciudad. Esta geografía de oportunidad (Galster et al., 2010; Hernández, 2012; Squires y Kubrin, 2005) ofrece la posibilidad de incrementar las oportunidades de trabajo, consumo o esparcimiento, si la movilidad lo permite, o la posibilidad de no acceder a estas oportunidades si la movilidad dificulta su acceso. El nivel de acceso de las personas a las distintas esferas de su vida cotidiana depende, entonces, tanto de la localización residencial, de la localización de estas esferas en la ciudad, como del capital de movilidad de las personas. De este modo, la accesibilidad a las distintas oportunidades que ofrece la ciudad 


\section{*.*vis revistainvi}

se determina por la disponibilidad de los variados modos de transporte y por las capacidades de las personas para acceder a ellos.

Las desigualdades urbanas en Santiago han estado marcadas por variados aspectos, pero han adquirido una manifestación contemporánea más clara, asociada a la segregación, desde la segunda mitad del siglo XX, al verificarse una fuerte migración campo-ciudad que determinó la localización de familias pobres en viviendas precarias en las periferias. Desde entonces, la pobreza y la desigualdad se hicieron visibles a través de la calidad y la localización de la vivienda.

El Plan Regulador Intercomunal de Santiago en 1960 intentó generar avances en materia de planificación urbana, pero el límite urbano establecido no resultó efectivo, siendo habitualmente sobrepasado por la urbanización de predios no urbanos y por las tomas de terrenos realizadas por pobladores sin casa (Gross, 1991).

A través del Decreto Supremo 420 de 1979, el gobierno de entonces buscó establecer una plena operación del mercado de suelo, al ampliar considerablemente el límite urbano, impulsando un modelo de carácter expansivo (Sabatini, 2000). Según Figueroa (2004, p. 249) la justificación invocada fue que "las regulaciones públicas afectan el mercado del suelo, transformándolo artificialmente en un bien escaso". Se esperaba así que la supresión del límite urbano produjera una mayor libertad para el mercado inmobiliario, incentivando un incremento en la disponibilidad de suelo y una disminución de precio. En definitiva, según el autor, el propósito consistía en introducir integralmente las leyes del mercado en el suelo urbano: además de la abolición del límite urbano se implementó el "realismo de precios", que significaba que la demanda efectiva solvente de las familias determinaba a qué suelo y localización tenía acceso cada persona según su nivel de ingreso. Ello fue materializado por el Estado a través de la erradicación de población pobre que residía en sectores de altos ingresos y el establecimiento de ley de municipalidades, que produjo una división comunal más fina, acorde a las condiciones de diferenciación socioeconómica entre comunas y de identidad social al interior de ellas, a finales de los años setenta.

Estos hechos marcaron paulatinamente la instalación de personas con menores ingresos en algunas comunas periféricas de la ciudad, en especial en el norte, sur y poniente. Las zonas residenciales de altos ingresos y el centro de negocios se expandieron hacia el oriente, principalmente en las comunas que tradicionalmente tenían un perfil socioeconómico más alto.

Desde la década de los ochenta y noventa, se produjo una mayor demanda pública y privada de terrenos periféricos, gracias a los bajos precios relativos de este suelo. De acuerdo a su localización, estos terrenos se utilizaron alternativamente para construir vivienda social o condominios residenciales de ingresos medios y altos. Este proceso se profundizó en el contexto económico expansivo de aquel período. Las consecuencias se manifestaron en desplazamientos cotidianos de mayores distancias y de tiempos (Escolano y Ortiz, 2007; Figueroa, 2004), perjudicando la calidad de vida en términos de accesibilidad al trabajo, servicios y equipamientos, así como la concentración de población pobre en zonas de peores condiciones socioeconómicas y de urbanización. 
La opción de la periferización y el acceso al automóvil privado en los niveles altos y medios tuvo una inusitada intensidad, en asociación con el crecimiento económico y las políticas liberales de uso del suelo y de importaciones. Esto significó un impulso al desarrollo inmobiliario en las periferias (López, 2005) y una fuerte consolidación de las empresas inmobiliarias dedicadas a la gran producción en serie de viviendas para estratos altos y medios. Fue así como los tiempos de viaje continuaron incrementándose y la accesibilidad perdiendo en calidad. Agostini et al. (2016) evidenciaron que la segregación se incrementó entre 1992 y 2002 en Santiago y confirmaron que en este período existió una profunda diferencia entre sus comunas, explicada por los cambios poblacionales y la expansión de la ciudad, sugiriendo que la segregación residencial se asocia a las dinámicas inmobiliarias y a la política de vivienda del período.

Cortés et al. (2016) consideran que los procesos de expansión urbana generan mayores tiempos de viaje, pero con gran diferencia según se use el automóvil privado o el transporte público. Dos factores adicionales apoyan la preferencia del vehículo privado: la comodidad del desplazamiento y lo barato de los vehículos; de acuerdo a estos autores, en Chile los automóviles tienen un precio final más bajo que en el resto de los países de la región latinoamericana. Sin embargo, no todos pueden acceder a su propiedad y a su uso, y la población de bajos ingresos es cautiva del transporte público. Del mismo modo, los usuarios del automóvil han mostrado, según los mismos autores, una inelasticidad muy alta frente al costo directo de su uso: a pesar de los incrementos en el precio del combustible o del peaje, no se verifica ni en el corto ni en el mediano plazo una reducción de la circulación en las calles de la ciudad ni en las autopistas concesionadas, sino, por el contrario, un aumento fluido.

Por otra parte, existe una importante población que no posee automóvil y que depende estrechamente de un transporte público de baja calidad, en especial, en las zonas más alejadas. Además, estos usuarios del transporte público tienen recursos limitados para su movilidad y deben aceptar un alto costo en tiempo para evitar gastar más en dinero líquido del cual no disponen; Herrera y Razmilic (2018) aseguran que existen tiempos excesivos de viaje al trabajo en Santiago, los que incluso son marcadamente más altos que en otras áreas metropolitanas del país, y que, igualmente existe una relación inversa entre el ingreso de los hogares y los tiempos de viaje. Ello se relaciona también con la oferta de transporte público, pues RED, (ex Transantiago, el sistema de transporte público de la ciudad) ha demostrado ser poco eficiente y muy oneroso para sus usuarios (Figueroa, 2013). 


\section{䌊 revistainvi}

\section{Metodología}

Dados los antecedentes mencionados hasta el momento, se consideran como variables a analizar el tiempo de viaje y su costo monetario directo. Estos son los principales componentes del denominado costo generalizado de transporte (Wingo, 1961).

De acuerdo a la información disponible, pero también por opción, se ha trabajado con los tiempos de viaje al trabajo, pues estos son los principales viajes regulares de las personas y los que están en el centro de la inserción social y económica de los individuos al momento de mayor concentración de viajes (horario punta mañana) y de mayor estrés sobre el sistema de transporte y de vialidad.

Esta variable permite además revelar distancias entre el lugar de residencia y los centros principales de trabajo, los cuales están concentrados principalmente en las comunas de Santiago, Providencia y Las Condes y dispersos en periferias industriales. Estas primeras zonas precisamente contienen los mayores precios de suelo, que trascienden a los usos residenciales dada la proximidad que tienen con servicios y equipamientos. Por lo tanto, personas con menores recursos tienen que asumir un costo de oportunidad en mayores tiempos de viaje a sus trabajos puesto que no pueden acceder a una vivienda cercana a estos centros laborales y de negocios.

Para investigar si los tiempos de viaje sirven efectivamente como variable explicativa y representativa para la mayoría de las desigualdades que existen en Santiago, se consultó la Encuesta de Caracterización Socio Económica (CASEN). Dicho instrumento, en su versión 2017, permite realizar un análisis metropolitano más actualizado, pero la versión 2015 tiene una información más completa y sólida, por lo que se ha combinado la información según el tema abordado. La versión 2015, dada su representatividad estadística comunal, permite el análisis de 26 comunas del Área Metropolitana de Santiago, las cuales son auto-representadas.

Cabe mencionar que, según esta encuesta, se registran disminuciones significativas en el porcentaje de personas en situación de pobreza por ingresos a nivel nacional, la cual pasa del $11,7 \%$ al 8,6\% entre 2015 y 2017 (Ministerio de Desarrollo Social y Familia, 2018a). No obstante, existe un fuerte patrón de desigualdad que se ha agudizado regularmente en la distribución del ingreso del último tiempo (con un GINI que avanzó a 0,501 en 2017, frente a 0,493 en 2015).

Con esta fuente se han calculado los tiempos de viaje al trabajo junto con otras variables socioeconómicas. La más evidente y global relación entre variables que puede explicar las desigualdades urbanas es la que relaciona los costos directos y tiempos de viaje con los ingresos monetarios del hogar. Este ingreso considera la suma de los ingresos autónomos (sueldos y salarios tanto monetarios como en especie) y los subsidios monetarios recibidos por todos los miembros del hogar (Ministerio de Desarrollo Social y Familia, 2018b).

En primer lugar se realizó un ejercicio, a escala metropolitana, sobre el peso de los costos mensuales de transporte en los ingresos del hogar según decil, a partir de distintas variables. 


\section{*.*vis revistainvi}

Inicialmente, a escala metropolitana y por decil, se obtuvieron el número promedio de personas por hogar, el ingreso promedio monetario del hogar, el número de veces en promedio que realiza el viaje al trabajo a la semana el (la) jefe(a) de hogar y los costos del pasaje o viaje del transporte público, considerando las tarifas de 2015 y 2017, que estaba fija entonces en 640 pesos chilenos en esos años (equivalente a 0,9 y 1,1 dólares americanos para esas fechas). Con estas variables se procedió a estimar los costos de los viajes semanales del jefe o jefa de hogar, de su pareja o segundo adulto y finalmente del resto de la familia. Para el o la jefe de hogar, se asumió (pues las encuestas solo muestran los viajes de ida al trabajo en los días laborales de la semana) que el doble de esta cifra corresponde al viaje con retorno, los cuales se multiplican por el costo del pasaje en hora punta, promediando entre el valor del viaje en bus y el de metro con combinación. Se consideran también cuatro viajes para el fin de semana (viajes de ocio y recreación) que toman el promedio del valor del pasaje fuera de punta entre el bus y el metro con combinación, y un viaje extra semanal con la tarifa en hora punta (probablemente para compras y trámites). Asimismo, se asume que una persona del grupo familiar (probablemente el segundo adulto) realiza todos los viajes entre semana menos dos (es decir un día a la semana no hace viajes), asumiendo una relación laboral más flexible y/o desplazamientos por otros motivos; en su caso, el gasto se obtiene considerando el valor del pasaje de hora punta en los viajes de ida, pero el valor del pasaje fuera de punta en los viajes de vuelta. Al igual que con el o la jefe de hogar, se asumen cuatro viajes para los fines de semana con la tarifa en horario no punta y un viaje extra semanal con la tarifa en hora punta. Para el resto del grupo familiar, se asume que realizan los mismos viajes que el primer viajero (probablemente, por motivo estudios) con tarifa de hora punta para los viajes de ida, pero con tarifa en horario no punta para los viajes de vuelta. Además, se consideran igualmente cuatro viajes con tarifa en horario no punta para los fines de semana y un viaje extra con la tarifa en horario punta.

Los costos semanales de viaje del grupo familiar se suman entre sí y se multiplican por cuatro, para obtener el costo de 30 días, que incluyen los viajes de 28 días que equivalen a cuatro semanas (viajes entre semana y de fines de semana) más los viajes de dos días adicionales (los viajes extra). Por último, se divide este costo total sobre los ingresos monetarios del hogar, para obtener el porcentaje que representa en cada decil. La siguiente formulación, sintetiza el procesamiento descrito:

$P C T_{i}=\frac{\left(\left(\left(v_{i} * 2 * c t_{p}+4 * c t_{f p}\right)+c t_{p}\right)+\left(\left(\left(v_{i}-1\right) *\left(c t_{p}+c t_{f p}\right)+4 * c t_{f p}\right)+c t_{p}\right)+\left(p_{i}-2\right) *\left(\left(v_{i} *\left(c t_{p}+c t_{f p}\right)+4 * c t_{f p}\right)+c t_{p}\right)\right) * 4}{I A H_{i}}$ 


\section{*.*vis revistainvi}

Donde:

- $\quad P C T_{i}$ es el peso del costo de viaje del hogar mensual sobre el ingreso monetario del hogar del decil i.

- $\quad v i$ es el número de veces en promedio que realiza el viaje al trabajo a la semana del decil $i$.

- $\quad c t_{p}$ es el costo del viaje en transporte público promedio en hora punta.

- $\quad c t_{f p}$ es el costo del viaje en transporte público promedio fuera de hora punta.

- $\quad p_{i}$ es el número promedio de personas por hogar del decil $i$.

- $\quad I_{A}$ es el ingreso promedio monetario del hogar del decil $i$.

En complemento con el análisis por condición socioeconómica, se ha trabajado con variables de localización. La homogeneidad de las comunas, dadas las consideraciones hechas más arriba, hace que la variable comunal sea una buena representación de no solo la localización, sino también del nivel de ingreso familiar en la ciudad. De esta manera, para esta dimensión se consideran los ingresos del hogar, el desempleo, el tiempo promedio de viaje y la motorización.

Si bien el uso de la Encuesta CASEN es uno de los principales aportes de esta investigación al ocupar las nuevas preguntas sobre movilidad que incorpora, este análisis se puede desarrollar también con la Encuesta Origen Destino (EOD). La EOD es un instrumento que interroga a una muestra de la población sobre sus prácticas de viaje, por lo que sirve para caracterizar los patrones de movilidad, motivos y frecuencias de los viajes que realiza esta población (Anapolsky, 2017). Para el caso del Gran Santiago, la última EOD se realizó en 2011-2012, razón por la cual se desestimó su uso en esta ocasión. No obstante, brinda antecedentes relevantes, dado que los viajes realizados en transporte público representaban en ese entonces el 29,1\% de la partición modal y los viajes al trabajo el 29,4\% del total (Universidad Alberto Hurtado, Observatorio Social, 2014). 


\section{Resultados}

Las referencias a los viajes al trabajo permiten deducir la movilidad del resto del grupo familiar, al que se le asigna desplazamientos equivalentes (pero minorados en cantidad y/o tarifa) debido a relaciones laborales menos formales (segundo adulto) o por causa de estudios para el resto. A partir de los datos de la encuesta CASEN que establecen una clasificación por deciles, identificando el número de personas del hogar, el ingreso promedio y la cantidad de viajes semanales al trabajo, se ha expandido la información al conjunto de la familia y al conjunto de los viajes realizados por todos. El peso del costo mensual del transporte público en el ingreso del hogar es el resultado de tal cálculo, el que se observa en la Tabla 1.

\section{Tabla 1.}

Peso del costo mensual del transporte público en el ingreso del hogar por deciles para la Región Metropolitana de Santiago.

\begin{tabular}{lllll} 
Decil & $\begin{array}{l}\text { Número } \\
\text { promedio de } \\
\text { personas por } \\
\text { hogar }\end{array}$ & $\begin{array}{l}\text { Ingreso promedio } \\
\text { monetario del hogar en } \\
\text { pesos chilenos }\end{array}$ & $\begin{array}{l}\text { Número de veces } \\
\text { promedio que realiza el } \\
\text { viaje a la semana }\end{array}$ & $\begin{array}{l}\text { Peso del costo mensual del } \\
\text { transporte público en el } \\
\text { ingreso del hogar }\end{array}$ \\
\hline I & 3,36 & $236.238,69$ & 5,12 & $55,77 \%$ \\
\hline II & 3,78 & $436.092,99$ & 5,39 & $35,41 \%$ \\
\hline III & 3,58 & $550.734,47$ & 5,13 & $25,57 \%$ \\
\hline IV & 3,40 & $642.311,95$ & 5,49 & $21,80 \%$ \\
\hline V & 3,49 & $812.825,75$ & 5,66 & $18,13 \%$ \\
\hline VI & 3,17 & $912.820,39$ & 5,43 & $14,17 \%$ \\
\hline VII & 2,94 & $1.064 .434,70$ & 5,41 & $11,20 \%$ \\
\hline VIII & 2,78 & $1.346 .634,70$ & 5,29 & $5,25 \%$ \\
\hline IX & 2,65 & $1.992 .729,20$ & 5,65 & $2,11 \%$ \\
\hline X & 2,32 & $4.237 .831,00$ & 5,08 & \\
\hline
\end{tabular}

Fuente: elaboración propia con base en CASEN 2017. 


\section{*.*vis revistainvi}

A escala metropolitana, se evidencian profundas desigualdades entre los deciles de ingreso de los hogares. Los costos mensuales del transporte público en el ingreso del hogar representan más del 55\% en el primer decil, mientras que, en el último, apenas supera el $2 \%$. No obstante, entre los hogares más ricos, por las características de su movilidad con tendencia hacia el automóvil privado, este valor es difícilmente comparable. Por lo que, en rigor, este trabajo se concentra preferentemente en los resultados de la población de menores ingresos. Entendiendo esto, el 10\% más pobre de los hogares tiene que gastar una suma de sus ingresos impracticable para su movilidad y entre los cinco deciles más pobres el gasto supera el 15\%, porcentaje que se ha subentendido entre expertos como un máximo razonable para las familias (Banco de Desarrollo de América Latina, 2010). En cambio, el 10\% de los hogares con mayores ingresos, tiene costos que son marginales considerando sus ingresos mensuales, aunque en este último caso se está suponiendo un hogar de altos ingresos viajando en transporte público, lo que no refleja bien la realidad.

Si se considera el gasto promedio de los hogares en transporte (aunque a nivel nacional), de acuerdo con la VIII Encuesta de Presupuestos Familiares (EPF) entre julio de 2016 y junio de 2017, este alcanza el 15,2\%, es decir, menos de lo que gastan los cinco quintiles más pobres de la ciudad, lo que es revelador de las profundas desigualdades socioeconómicas y además demostrativo de la gravedad de la incidencia de los costos de transporte entre la población más pobre.

Realizando el mismo ejercicio a escala comunal, es decir, asociado a la localización residencial, se aprecia que las desigualdades también son evidentes. Aquí el peso promedio del costo mensual del transporte público en el ingreso del hogar tiende a aparecer atenuado debido a que se consideran solo promedios que esconden la realidad, pues existen no solo grandes diferencias entre las comunas que conforman la Región Metropolitana sino también al interior de la mayoría de ellas. Por encima del 15\% de costo promedio comunal, que, como se ha visto, es la referencia habitualmente considerada como un máximo aceptable de este costo, se encuentran 16 comunas como La Pintana, Cerro Navia, El Bosque, San Bernardo y Colina, en contraste con Ñuñoa, Las Condes y Providencia que gastan menos del $6 \%$ y otras siete que se encuentran entre $14,7 \%$ y $8,3 \%$. Como se aprecia en la Tabla 2, la mayoría de las comunas presentan entre un $10 \%$ y un $23,5 \%$ de la incidencia de los costos mensuales de transporte público en el ingreso del hogar, y solo cinco en ellas gasta en promedio menos del $10 \%$. 


\section{* revistainvi}

Tabla 2.

Peso del costo mensual del transporte público en el ingreso del hogar por comunas para la Región Metropolitana.

\begin{tabular}{|c|c|c|c|c|}
\hline Comuna & $\begin{array}{l}\text { Número promedio } \\
\text { de personas por } \\
\text { hogar }\end{array}$ & $\begin{array}{l}\text { Ingreso promedio } \\
\text { monetario del hogar en } \\
\text { pesos chilenos }\end{array}$ & $\begin{array}{l}\text { Número de veces promedio } \\
\text { que realiza el viaje a la } \\
\text { semana }\end{array}$ & $\begin{array}{l}\text { Peso del costo mensual de } \\
\text { transporte público en el } \\
\text { ingreso del hogar }\end{array}$ \\
\hline La Pintana & 3,97 & $664.811,30$ & 5,16 & $23,5 \%$ \\
\hline Cerro Navia & 3,49 & $620.550,76$ & 5,29 & $22,5 \%$ \\
\hline El Bosque & 3,56 & $653.960,91$ & 5,04 & $21,0 \%$ \\
\hline San Bernardo & 3,38 & $758.384,99$ & 6,44 & $20,5 \%$ \\
\hline Colina & 3,58 & $681.916,82$ & 5,06 & $20,3 \%$ \\
\hline Peñalolén & 3,74 & $706.539,60$ & 4,89 & $20,1 \%$ \\
\hline Renca & 3,50 & $677.747,91$ & 5,04 & $19,9 \%$ \\
\hline Conchalí & 3,45 & $711.382,77$ & 4,99 & $18,6 \%$ \\
\hline Melipilla & 3,13 & $662.250,34$ & 5,16 & $18,5 \%$ \\
\hline Recoleta & 3,21 & $676.489,64$ & 5,05 & $18,3 \%$ \\
\hline Pudahuel & 3,39 & $742.253,43$ & 5,13 & $17,8 \%$ \\
\hline La Granja & 3,59 & $767.051,13$ & 4,92 & $17,8 \%$ \\
\hline Estación Central & 3,11 & $705.931,25$ & 5,06 & $17,0 \%$ \\
\hline Quilicura & 3,62 & $890.655,12$ & 5,28 & $16,2 \%$ \\
\hline Talagante & 3,30 & $824.971,11$ & 5,40 & $16,2 \%$ \\
\hline Puente Alto & 3,60 & $854.073,07$ & 4,98 & $16,1 \%$ \\
\hline Maipú & 3,54 & $965.511,37$ & 5,33 & $14,7 \%$ \\
\hline La Florida & 3,35 & $931.329,19$ & 4,95 & $13,7 \%$ \\
\hline Quinta Normal & 2,78 & $786.455,79$ & 5,08 & $13,6 \%$ \\
\hline $\begin{array}{l}\text { Pedro Aguirre } \\
\text { Cerda }\end{array}$ & 3,58 & $1.138 .246,60$ & 5,04 & $12,1 \%$ \\
\hline Macul & 3,35 & $1.188 .637,00$ & 4,86 & $10,6 \%$ \\
\hline San Miguel & 3,08 & $1.507 .958,60$ & 5,55 & $8,4 \%$ \\
\hline Santiago & 2,29 & $1.047 .740,00$ & 5,06 & $8,3 \%$ \\
\hline Ñuñoa & 2,63 & $1.858 .190,80$ & 4,88 & $5,3 \%$ \\
\hline Las Condes & 2,98 & $2.918 .799,50$ & 4,78 & $3,8 \%$ \\
\hline Providencia & 2,27 & $2.567 .129,70$ & 4,94 & $3,3 \%$ \\
\hline
\end{tabular}

Fuente: elaboración propia con base en CASEN 2015 (Ministerio de Desarrollo Social y Familia, 2015). 


\section{粼 revistainvi}

La distribución del ingreso territorial muestra con claridad una estructura desigual y segregada según la localización relativa en la ciudad. Mientras las comunas más ricas están concentradas en el oriente (Las Condes, Providencia, Ñuñoa), algunas que poseen menores recursos se localizan casi en el límite urbano (El Bosque, La Pintana, Cerro Navia) o fuera de este (Colina, Melipilla), lo que puede apreciarse en la Figura 1.

\section{Figura 1.}

Ingreso promedio monetario del hogar en pesos chilenos por comuna de la Región Metropolitana.

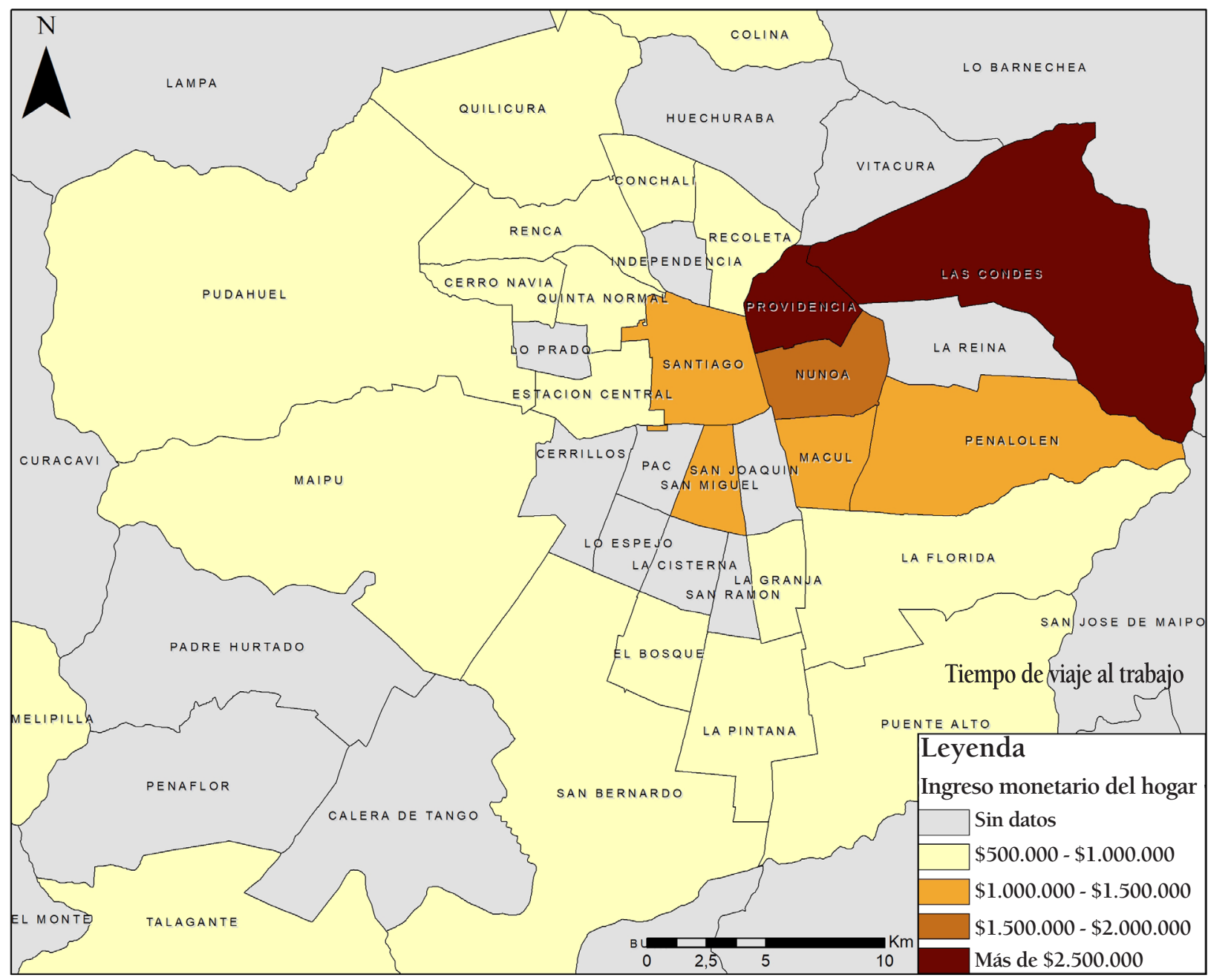

Fuente: elaboración propia con base en CASEN 2015 (Ministerio de Desarrollo Social y Familia, 2015). 
La Tabla 3 muestra la relación de los tiempos de viaje promedio al trabajo por comuna. Representan solo un viaje de ida por lo que el total del desplazamiento ida y vuelta correspondería a un valor en torno al doble de ese tiempo.

Tabla 3.

Tiempo de viaje promedio al trabajo por comuna de la Región Metropolitana.

\begin{tabular}{ll} 
Comunas & Tiempo de viaje promedio al trabajo en minutos \\
\hline Providencia & 31,62 \\
\hline Las Condes & 34,48 \\
\hline Santiago & 39,87 \\
\hline Nuñoa & 40,08 \\
\hline Melipilla & 40,18 \\
\hline San Miguel & 42,45 \\
\hline Recoleta & 44,76 \\
\hline Pedro Aguirre Cerda & 44,97 \\
\hline Colina & 45,96 \\
\hline Estación Central & 46,15 \\
\hline Quinta Normal & 46,82 \\
\hline Talagante & 47,80 \\
\hline Conchalí & 47,91 \\
\hline Macul & 49,03 \\
\hline La Granja & 50,93 \\
\hline Quilicura & 51,37 \\
\hline Peñalolén & 52,43 \\
\hline Pudahuel & 53,88 \\
\hline La Florida & 55,13 \\
\hline Renca & 56,26 \\
\hline El Bosque & 58,04 \\
\hline Cerro Navia & 58,96 \\
\hline Maipú & 59,08 \\
\hline Puente Alto & 60,38 \\
\hline 61,75 \\
\hline 61,94 \\
\hline Pantana & \\
\hline
\end{tabular}

Fuente: elaboración propia con base en CASEN 2015 (Ministerio de Desarrollo Social y Familia, 2015). 


\section{*.*vis revistainvi}

Se aprecia que los menores valores (de 30 a menos de 40 minutos) se encuentran en las comunas de Las Condes, Providencia y Santiago, que tienen ingresos más altos y evidencian una alta concentración de actividades propias de la centralidad en sus territorios, como fuerte concentración empresarial, de servicios y comercios. Le siguen en tiempo las comunas pericentrales, por su cercanía, con tiempos de viaje al trabajo de entre 40 y 50 minutos. En contraste, otras tres comunas con estos tiempos promedio de viaje se localizan en las afueras de la mancha urbana (Talagante, Melipilla y Colina), lo que se explica igualmente porque estas comunas separadas del Gran Santiago tienen una importante fracción de actividades autónomas, y en el interior de ellas se concentra una buena parte de los destinos de trabajo de sus residentes.

El grupo de comunas que se encuentra al exterior de la circunvalación Américo Vespucio y dentro del límite urbano posee tiempos de viaje promedio entre 50 minutos y una hora. No obstante, las comunas con peores tiempos de viaje promedio son las que se localizan al sur de la ciudad (San Bernardo, La Pintana, Puente Alto) y tienen ingresos relativamente bajos.

A continuación, La Figura 2 pone en evidencia gráficamente el análisis desarrollado arriba y las relaciones espaciales. 


\section{* revistainvi}

\section{Figura 2.}

Tiempo de viaje promedio al trabajo en minutos por comuna de la Región Metropolitana.

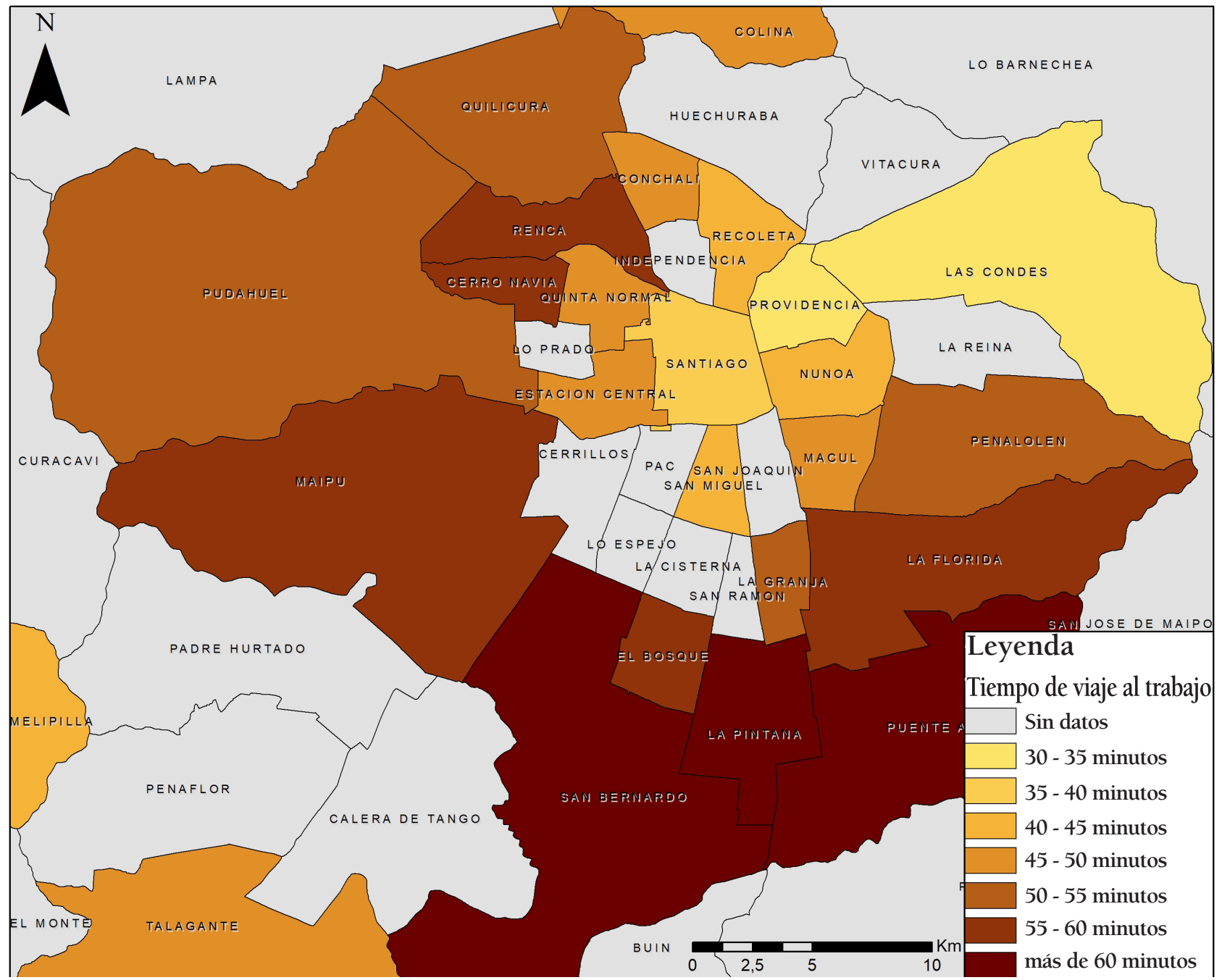

Fuente: elaboración propia con base en CASEN 2015 (Ministerio de Desarrollo Social y Familia, 2015). 
Se puede confirmar aquí que las comunas del sector nororiente de la ciudad, el de mayor ingreso familiar relativo (Las Condes y Providencia), poseen el menor tiempo de viaje promedio al trabajo, seguidas por la comuna central de Santiago. Entre los 40 y 50 minutos, se encuentran las comunas de las zonas norte (Recoleta, Conchalí y Quilicura) y fuera del área urbana consolidada (Colina, Melipilla y Talagante). Todas las comunas de las zonas norponiente (Cerro Navia, Renca, Quinta Normal y Pudahuel), sur (La Pintana, El Bosque, San Bernardo, Pedro Aguirre Cerda, La Granja y San Miguel), suroriente (Puente Alto, La Florida, Peñalolén y Macul) y surponiente (Estación Central y Maipú), superan los 50 minutos de tiempo de viaje promedio al trabajo.

La Tabla 4 muestra la relación entre el ingreso promedio de zonas agregadas de la ciudad y los tiempos de viaje. Se puede apreciar aquí que el efecto distancia tiene un rol efectivo en la definición de los tiempos de viaje, reforzando en muchos casos la tendencia de la condición socioeconómica. Sin embargo, no debe olvidarse que, como ya se ha visto aquí, el hábitat de los mayores ingresos es por definición uno de mejor accesibilidad.

\section{Tabla 4.}

Ingreso promedio monetario del hogar en pesos chilenos y tiempo de viaje promedio al trabajo por zonas para la Región Metropolitana.

\begin{tabular}{lll} 
Zona & $\begin{array}{l}\text { Ingreso promedio monetario } \\
\text { del hogar en pesos chilenos }\end{array}$ & $\begin{array}{l}\text { Tiempo de viaje promedio al } \\
\text { trabajo }\end{array}$ \\
\hline Centro & $1.047 .740,00$ & 39,87 \\
\hline Nororiente & $2.448 .040,00$ & 35,39 \\
\hline Norponiente & $706.751,97$ & 53,98 \\
\hline Norte & $759.509,18$ & 48,01 \\
\hline Comunas fuera del área urbana & $723.046,09$ & 44,65 \\
\hline consolidada & $915.068,92$ & 53,09 \\
\hline Sur & $920.144,72$ & 54,63 \\
\hline Suroriente & $835.721,31$ & 52,61 \\
\hline Surponiente & $1.019 .575,72$ & 49,32 \\
\hline Total general &
\end{tabular}

Fuente: elaboración propia con base en CASEN 2015 (Ministerio de Desarrollo Social y Familia, 2015). 


\section{revistainvi}

Al relacionar a nivel comunal el ingreso promedio monetario del hogar con el tiempo de viaje al trabajo se evidencian también importantes desigualdades territoriales que confirman fehacientemente el análisis anterior. Existe una relación inversa entre ingreso y tiempos de viaje, como se observa en la Figura 3.

\section{Figura 3.}

Ingreso promedio monetario del hogar en pesos chilenos y tiempo de viaje promedio al trabajo por comuna de la Región Metropolitana.

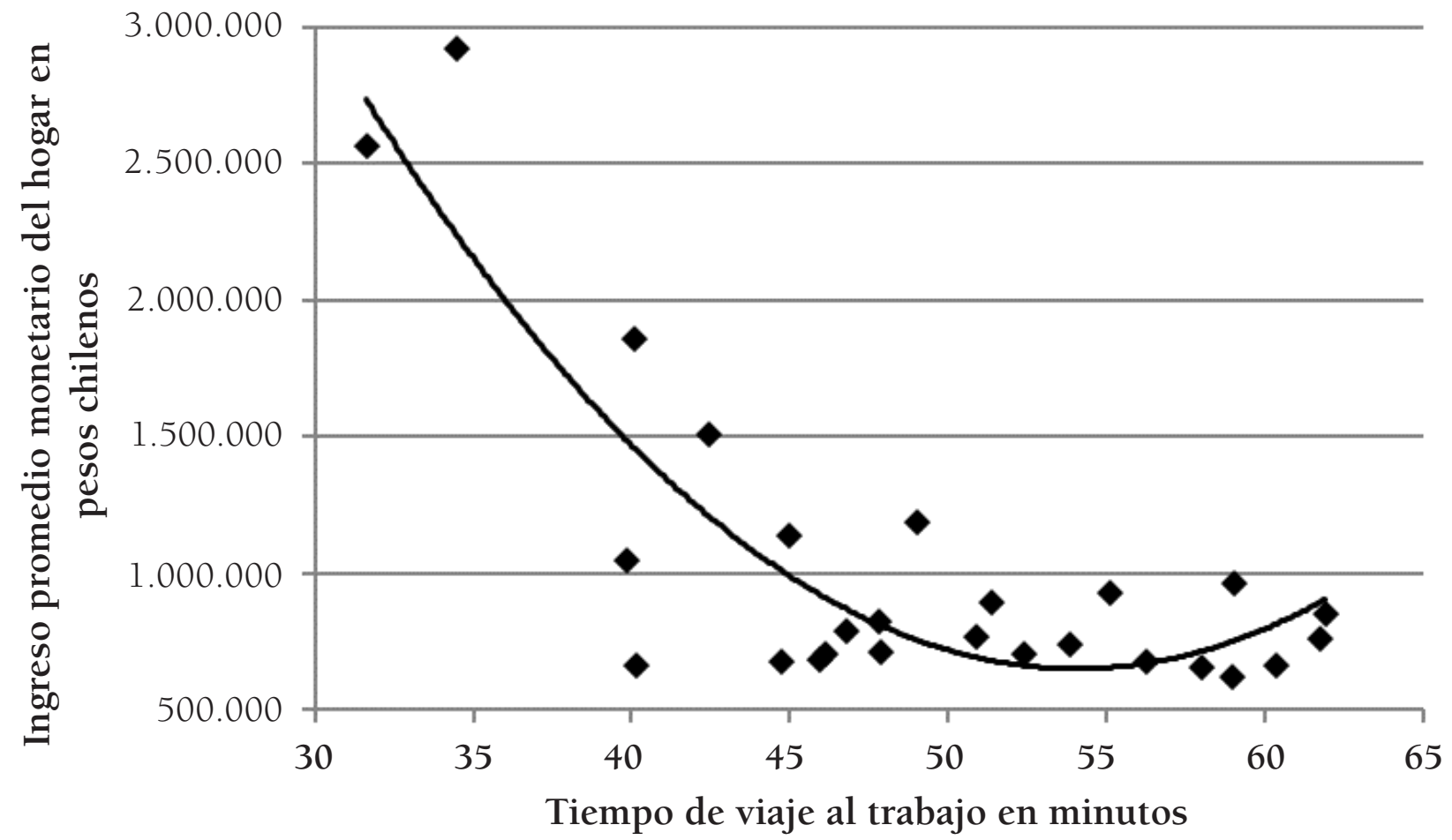

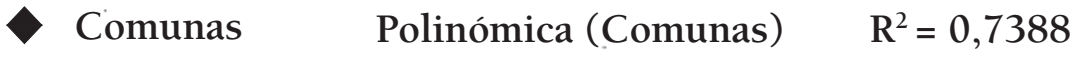

Fuente: elaboración propia con base en CASEN 2015 (Ministerio de Desarrollo Social y Familia, 2015). 


\section{*.*vis revistainvi}

Esta figura es muy clara en mostrar la relación directa entre tiempos de viaje y condición socioeconómica. Las comunas del oriente invierten el menor tiempo en los viajes al trabajo, entre otras cosas porque siendo los sectores de mayores ingresos, han desarrollado sus zonas con la mejor accesibilidad en la ciudad, y además ejercen una fuerza gravitatoria en el desarrollo urbano, promoviendo la expansión del centro hacia las cercanías de sus zonas de residencia. Al otro lado, se encuentran las comunas de San Bernardo y Puente Alto con mayores tiempos promedio de viaje (más de 60 minutos) y un porcentaje de pobreza relativamente alto (cerca al 10\%, CASEN 2015). Sin embargo, El Bosque y La Pintana presentan un mayor porcentaje de pobreza (entre el 12\% y 15\%) y un tiempo de viaje próximo a los 60 minutos. También se dan casos atípicos a este comportamiento, como por ejemplo las comunas de Recoleta y Colina, con un nivel de pobreza de 14\% y unos tiempos promedio de viaje de alrededor de 45 minutos, que se explican, en el caso de Recoleta, por su cercanía a las comunas centrales, y en el caso de Colina, por una importante proporción de hogares de altos ingresos que se instalaron allí.

Al analizar los tiempos de viaje y las tasas de desempleo por comuna, se evidencia una correlación más moderada. Sin embargo, es posible constatar la relación existente entre localización residencial y oportunidades laborales: según los datos de CASEN (2015), La Pintana, Puente Alto y San Bernardo se ubican en el grupo de comunas del extremo con mayor desempleo (más del 8\%) y mayores tiempos de viaje.

Por último, las condiciones de accesibilidad en tiempo y costo monetario están también fuertemente vinculadas a la disponibilidad de medios de transporte de los ciudadanos. Considerando la información de la CASEN 2015, se observa en la Tabla 5 que las comunas que tienen mayores ingresos y menores tiempos de viaje poseen una mayor tasa de motorización. 
Tabla 5 .

Tasa de motorización y vehículos por cada 1.000 habitantes por comuna de la Región Metropolitana.

\begin{tabular}{|c|c|c|}
\hline Comuna & Tasa de motorización (vehículos/hogares) & Vehículos por cada 1000 habitantes \\
\hline Las Condes & 1,06 & 348 \\
\hline Providencia & 0,69 & 300 \\
\hline Ñuñoa & 0,67 & 253 \\
\hline San Miguel & 0,57 & 186 \\
\hline Macul & 0,53 & 158 \\
\hline Maipú & 0,53 & 149 \\
\hline Peñalolén & 0,44 & 119 \\
\hline Puente Alto & 0,43 & 121 \\
\hline La Florida & 0,4 & 120 \\
\hline Quilicura & 0,4 & 110 \\
\hline Talagante & 0,39 & 117 \\
\hline Quinta Normal & 0,36 & 129 \\
\hline La Granja & 0,33 & 91 \\
\hline Melipilla & 0,33 & 106 \\
\hline San Bernardo & 0,33 & 98 \\
\hline Pudahuel & 0,32 & 95 \\
\hline Estación Central & 0,29 & 93 \\
\hline Renca & 0,29 & 83 \\
\hline Colina & 0,28 & 77 \\
\hline Conchalí & 0,28 & 82 \\
\hline El Bosque & 0,27 & 76 \\
\hline Santiago & 0,25 & 111 \\
\hline Cerro Navia & 0,23 & 66 \\
\hline Pedro Aguirre Cerda & 0,23 & 65 \\
\hline Recoleta & 0,21 & 65 \\
\hline La Pintana & 0,19 & 48 \\
\hline
\end{tabular}

Fuente: elaboración propia con base en CASEN 2015 (Ministerio de Desarrollo Social y Familia, 2015). 


\section{*.*vis revistainvi}

Las tasas de propiedad de vehículos según comunas son rotundas para confirmar que existe una fuerte relación entre las condiciones de movilidad y la propiedad de vehículos motorizados. Las comunas más ricas de la ciudad exhiben fuertes diferencias en este ámbito respecto a las más pobres. En Chile es habitual que los propietarios de automóviles se sirvan intensamente de su vehículo, pero una vez más esta situación está cruzada por el ingreso. Según Landon (2016), la población de menores ingresos que posee automóvil restringe su uso por varios motivos: los vehículos de su propiedad son antiguos y a veces están en mal estado, por lo que su uso implica riesgos de desperfectos en el camino; debido al nivel de ingreso se trata de población sensible al costo de la gasolina y también y en mayor medida, al costo de los peajes de las autopistas urbanas; por último, estos automóviles a menudo no tienen sus papeles en regla.

\section{Discusión}

La investigación evidencia la fuerte correlación que existe entre las condiciones socioeconómicas de la población y su accesibilidad, medida en tiempos y en costos monetarios directos de viaje.

En el caso específico del Área Metropolitana de Santiago, las oportunidades se concentran en la zona oriente de más altos ingresos. Esta situación refuerza aún más el rol clave de la movilidad en la accesibilidad de las personas a las áreas de oportunidades (Cerda y Marmolejo, 2010). En ese contexto, los sectores más vulnerables deben enfrentar dos escenarios de movilidad cotidiana: recorrer las distancias necesarias para alcanzar las áreas de oportunidades o simplemente renunciar a desplazarse y buscar alternativas más escasas o menos interesantes, pero más locales (Prieto y Brain, 2018).

Se aprecia de qué manera las condiciones de localización poblacional coinciden igualmente con las condiciones socioeconómicas, es decir, que existe una opción de localización residencial (y de sus trabajos correspondientes) privilegiada para los ricos que potencia aún más su accesibilidad mientras que para la población de menores ingresos sucede lo contrario. Del mismo modo, la propiedad de automóviles y la situación laboral en las distintas comunas de Santiago refuerzan aún más esta condición. Por una parte, las comunas del pericentro, que habitualmente en las ciudades tienen un grado de pobreza importante, se benefician de la cercanía de las zonas centrales y sus tiempos de viaje entonces son menores; por otra parte, algunas comunas distantes, aunque sean pobres generan sus propias fuentes de trabajo, en especial aquellas no conurbadas. Entre las dos situaciones, existen casos de comunas con ausencia de fuentes de trabajo, que obligan a sus vecinos a hacer largos viajes, y otras donde no existen oportunidades y el costo del transporte promueve la inmovilidad; por lo que se nutren de trabajos locales, muy precarios y artesanales (ver, por ejemplo, el análisis de Jouffe, 2010).

Con ello, se aprecian claramente las desigualdades en el ámbito de la movilidad y la accesibilidad para los distintos estratos de población. Si bien los costos de transporte se calcularon para el uso del transporte público, principal opción de transporte para los hogares de menores ingresos, la preferencia por el automóvil privado 


\section{*.*vis revistainvi}

en las comunas de medios y altos ingresos ofrece mejores condiciones tanto en tiempos como en calidad de los viajes.

De acuerdo a Cortés et al. (2016), esta preferencia no pareciera tender a cambiar al menos en el corto o mediano plazo. Ad portas de una nueva licitación del sistema RED (ex Transantiago) tras más de 10 años de graves fallas financieras y operativas del sistema, la implementación de nuevas líneas de metro y la no disponibilidad de nuevos modos de transporte sustentables de arriendo (bicicletas y scooters eléctricas) en ciertas comunas, las expectativas sobre la movilidad metropolitana son definitivamente diversas y están segmentadas según la condición socio económica.

En la medida que la accesibilidad está en la base de las oportunidades disponibles para asegurar su bienestar (Garretón, 2011), el costo monetario de la movilidad para el 10\% más pobre de los hogares los condena a la perpetuación de la condición actual. Es probable que desde este grupo salga un buen porcentaje de los viajeros que practican la evasión en el sistema RED, o que simplemente no trabajen por no poder viajar o que opten por trabajos precarios cerca de su domicilio.

Aunque la relación entre los tiempos de viaje y las tasas de desempleo por comuna está menos desarrollada en este trabajo, para un mayor entendimiento de ella se puede acudir al trabajo de Herrera y Razmilic (2018) que muestran que a nivel metropolitano existen mayores tiempos promedio de viaje para "Personal doméstico y afines, Limpiadores, Lavanderos y Planchadores", y "Oficiales y Operarios de la Construcción (Obra Gruesa) y afines", con 70 y 60 minutos respectivamente. Los trabajadores de estas actividades viven habitualmente en el sur y norte pobres de la ciudad.

Por último, puede apreciarse también que los procedimientos determinantes llevados a cabo durante los años 80 del siglo pasado, de mercantilizar el suelo, reorganizar la población en función de nivel de ingreso y dividir la ciudad en comunas homogéneas desde el punto de vista socioeconómico, han tenido un gran éxito en perpetuar y potenciar estas condiciones de desigualdad, segregación e inequidad.

\section{Conclusiones}

La investigación ha permitido corroborar la hipótesis inicial. Efectivamente, en el Gran Santiago, los mayores costos de viaje (en tiempo y costo monetario) se asocian a condiciones socioeconómicas del territorio altamente segregado, y se concentran en la población de más bajos ingresos y zonas aparatadas y desfavorecidas, reproduciendo dinámicas de segregación y desigualdad socio espacial.

Como se ha visto, el costo de transporte pesa mucho más en el presupuesto de los hogares de categorías socioeconómicas más desfavorecidas que en el resto. En el caso específico del Área Metropolitana de Santiago, se suman a estos costos monetarios, bajo la misma lógica, los tiempos de viajes más extensos que dificultan 


\section{*.* revistainvi}

aún más el acceso a la geografía de oportunidades que ofrece una ciudad altamente centralizada en tres o cuatro comunas del sector oriente.

La organización urbana existente, determinada por costos de suelo y economías de urbanización que concentran los mercados laborales, configura inflexiblemente los patrones de localización residencial y movilidad. En otras palabras, se vuelven variables cotidianas que influencian las decisiones y estrategias de las personas, exigiéndoles definir costos de oportunidad en la elección de lugar de residencia y mayores o menores tiempos de viaje al trabajo. Dichos costos de oportunidad, que se ven limitados por el ingreso en cuanto a la posibilidad de ejercer una libertad de elección, a su vez interiorizan los problemas históricos de la planificación urbana, como la falta de articulación entre la planificación de la vivienda y la infraestructura de transporte.

El análisis aquí presentado pone en evidencia no solo un resultado puntual y estático, sino que revela las condiciones de la persistencia y reproducción de esta condición. En efecto, el costo de la movilidad y su peso en los presupuestos familiares se transforma en un lastre que reduce aún más el acceso a las oportunidades para los más pobres. De esta forma, su accesibilidad castigada no podrá ser un factor de solución de la pobreza, sino que la reproducirá regularmente; lo que convierte al costo de transporte y su peso en los presupuestos pobres en un factor de persistencia de tal condición.

Es así como el costo de la movilidad se vuelve factor crítico para las familias pobres. Frente a las desigualdades de acceso a las oportunidades para los sectores más desfavorecidos, la movilidad es en rigor sinónimo de obligación impuesta del cotidiano. En el año 2019, el aumento del pasaje de transporte público -y el rechazo a esto manifestado por la ciudadanía- fueron los hechos particulares que resultaron en el estallido social. Aunque este rango de incremento de tarifas pareciera ser menor, para la población más vulnerable esos 30 pesos adicionales de más de pasaje (en torno a los 4,5 centavos de dólar americano) representaron una fuerte y constante presión sobre el presupuesto del hogar, dificultando aún más su capacidad de movilidad y su acceso a oportunidades. El rechazo al aumento del pasaje puso así en el debate público la fuerte relación que existe entre el costo de la movilidad y las desigualdades sociales urbanas.

En el contexto de la pandemia de Covid-19, estas dinámicas de desigualdades urbanas asociadas a la movilidad se han profundizado. Se visibilizó aún más la brecha social y los fenómenos de segregación socio espacial, agravando probablemente las condiciones de accesibilidad de los más pobres. En este contexto, la población más vulnerable se enfrenta a un dilema fundamental: optar por el uso del transporte público para poder acceder a las fuentes de ingresos (que usualmente requieren de presencialidad) aumentando así la exposición al contagio; u optar por quedarse en casa, disminuyendo así la probabilidad de adquirir el virus, pero quedando sin acceso a alguna fuente cercana de trabajo y, por lo tanto, sin ingresos. 


\section{Referencias bibliográficas}

Agostini, C., Hojman, D., Román, A., y Valenzuela, L. (2016). Segregación residencial de ingresos en el Gran Santiago, 1992-2002: una estimación robusta. EURE, 42(127), 159-184. https://doi.org/10.4067/ $\underline{\text { S0250-71612016000300007 }}$

Alonso, W. (1964). Location and land use. Toward a general theory of land rent. Harvard University Press.

Anapolsky, S. (2017). Encuesta de origen y destino. En A. Gutiérrez, (Coord.). Manual sobre metodologías de estudio aplicables a la planificación y gestión del transporte y la movilidad: recomendaciones sobre el uso de herramientas cuali-cuantitativas de base territorial. (pp. 1724). Eudeba.

Ascher, F. (1995). Métapolis ou l'avenir des villes. Odile Jacob.

Banco de Desarrollo de América Latina. (2010). Observatorio de movilidad urbana para América Latina. http:// scioteca.caf.com/handle/123456789/420

Bauman, Z. (2000). Liquid modernity. Polity

Cerda, J. y Marmolejo, C. (2010). De la accesibilidad a la funcionalidad del territorio: una nueva dimensión para entender la estructura urbano-residencial de las áreas metropolitanas de Santiago (Chile) y Barcelona (España). Revista de Geografía Norte Grande, (46), 5-27. https://doi.org/10.4067/S0718-34022010000200001

Cortés, A., Figueroa, Ó. y Moreno, D. (2016). Los costos del uso del automóvil y su elasticidad. El caso de Santiago de Chile. Estudios Socioterritoriales, Revista de Geografía, (20), 27-39.

Escolano, S. y Ortiz, J. (2007). Patrones espaciales de movilidad de la población: algunos efectos en la sociogeografía del gran Santiago. En C. d. Mattos y R. Hidalgo, Santiago de Chile, movilidad espacial y reconfiguración metropolitana. Pontificia Universidad Católica de Chile.

Figueroa, ó. (2004). Infraestructura, servicios públicos y expansión urbana en Santiago. En C. d. Mattos, M. E. Ducci, A. Rodríguez, G. Yáñez, Gloria (Eds.) (2004) Santiago en la globalización zuna nueva ciudad? SUR.

Figueroa, Ó. (2013). Four decades of changing transport policy in Santiago, Chile. Research in Transportation Economics, 40(1), pp. 87-95.https://doi.org/10.1016/j. retrec.2012.06.031

Galster, G., Andersson, R., y Musterd, S. (2010). Who is affected by neighbourhood income mix? Gender, age, family, employment and income differences. Urban Studies, 47(14), 2915-2944. https://doi. org/10.1177/0042098009360233

Garretón, M. (2011). Desigualdad espacial y utilidad social: esfuerzos de movilidad y accesibilidad en el Gran Santiago. Revista Territorios, (25), 35-64.

Gross, P. (1991). Santiago de Chile (1925-1990): planificación urbana y modelos políticos. EURE, 17(52-53), 27-52.

Hernández, D. (2012). El transporte público y los desafíos del bienestar. Movilidad y accesibilidad en sectores populares de la ciudad de Montevideo. (Tesis doctoral, sin publicar). Universidad Católica de Chile, Santiago, Chile.

Herrera, A. y Razmilic, S. (2018). Movilidad urbana: Santiago no es Chile. Puntos de Referencia, (475).

Jirón, P., Lange, C., y Bertrand, M. (2010). Exclusión y desigualdad espacial: Retrato desde la movilidad cotidiana. Revista INVI, 25(68), 15-57. https://doi. org/10.4067/S0718-83582010000100002 
Jirón, P., y Mansilla, P. (2014). Las consecuencias del urbanismo fragmentador en la vida cotidiana de habitantes de la ciudad de Santiago de Chile. EURE, 40(121), 5-28. https://doi.org/10.4067/ $\underline{\text { S0250-71612014000300001 }}$

Jouffe, Y. (2010). ¿Derecho a la ciudad accesible o a la ciudad cercana? Discusión de una reivindicación ambigua desde el caso de hogares pobres de la periferia de Santiago de Chile. Actas del XI Coloquio Internacional de Geocrítica: La planificación territorial y el urbanismo desde el diálogo y la participación, Buenos Aires, Argentina.

Kaufmann, V. (2008). Les paradoxes de la mobilité. Presses Polytechniques et Universitaires Romandes.

Landon, P. (2016). Prácticas de movilidad cotidiana de las familias que habitan en barrios intervenidos por la autopista Acceso Sur en la ciudad de Santiago de Chile (Tesis de Doctorado Arquitectura y Estudios Urbanos, sin publicar). Pontificia Universidad Católica de Chile, Santiago, Chile).

López, E. (2005). Impacto del crecimiento del Gran Santiago en el deterioro funcional de sus espacios pericentrales. Scripta Nova. Revista electrónica de geografía y ciencias sociales, 9(194(47)).

Lucas, K. (2012). Transport and social exclusion: Where are we now? Transport Policy, 20, 105- 113. https:// doi.org/10.1016/j.tranpol.2012.01.013

Ministerio de Desarrollo Social y Familia. (2015). Encuesta de caracterización socioeconómica nacional CASEN 2015. http://observatorio.ministeriodesarrollosocial. gob.cl/encuesta-casen-2015

Ministerio de Desarrollo Social y Familia. (2018a). Situación de pobreza. Sintesis de resultados 2017. http://observatorio.ministeriodesarrollosocial.gob.cl/storage/ docs/casen/2017/Resultados pobreza Casen 2017. $\underline{\mathrm{pdf}}$
Ministerio de Desarrollo Social y Familia. (2018b). Informe de desarrollo social 2018. https://www. desarrollosocialyfamilia.gob.cl/storage/docs/Informe_de_Desarrollo Social 2018.pdf

Preston, J. (2009). Epilogue: Transport policy and social exclusion-Some reflections. Transport Policy, 16(3), 140-142. https://doi.org/10.1016/j. tranpol.2009.04.003

Prieto, J. y Brain, I. (2018). Movilidad cotidiana y cambios en la geografía de oportunidades en la ciudad de Santiago: ¿Qué ha pasado con las familias que viven mal localizadas? Lincoln Institute of Land Policy.

Sabatini, F. (2000). Reforma de los mercados de suelo en Santiago, Chile: efectos sobre los precios de la tierra y la segregación residencial. EURE, 26(77), 49-80. https://doi.org/10.4067/S0250-71612000007700003

Sheller, M. (2018). Mobility justice. Verso.

Sheller, M. y Urry, J. (2006). The new mobilities paradigm. Environment and Planning A: Economy and Space, 38(2), 207-226. https://doi.org/10.1068\%2Fa37268

Squires, G. D. y Kubrin, C. E. (2005). Privileged places: Race, uneven development and the geography of opportunity in urban America. Urban Studies, 42(1), 47-68. https://doi.org/10.1080/0042098042000309694

Universidad Alberto Hurtado, Observatorio Social. (2014). Actualización y recolección de información del sistema de transporte urbano, IX Etapa: Encuesta Origen Destino Santiago 2012. Encuesta origen destino de viajes 2012 (Documento Difusión). Sectra.

Wenglenski, S. (2010). Accès à l'emploi: Champs des possibles contre champs des pratiques. En M. Massot (Ed.) Mobilité \& modes de vie métropolitains (pp. 121137). L'Oeil d'or.

Wingo, L. (1961). Transportation and urban land. RFF Press. 


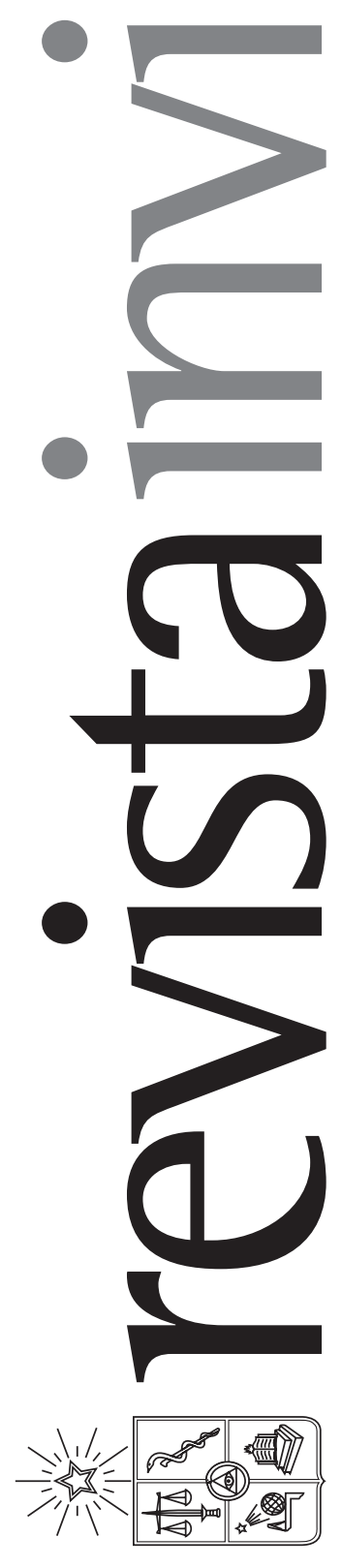

Revista INVI es una publicación periódica, editada por el Instituto de la Vivienda de la Facultad de Arquitectura y Urbanismo de la Universidad de Chile, creada en 1986 con el nombre de Boletín INVI. Es una revista académica con cobertura internacional que difunde los avances en el conocimiento sobre la vivienda, el hábitat residencial, los modos de vida y los estudios territoriales. Revista INVI publica contribuciones originales en español, inglés y portugués, privilegiando aquellas que proponen enfoques inter y multidisciplinares y que son resultado de investigaciones con financiamiento y patrocinio institucional. Se busca, con ello, contribuir al desarrollo del conocimiento científico sobre la vivienda, el hábitat y el territorio y aportar al debate público con publicaciones del más alto nivel académico.

Directora: Dra. Mariela Gaete Reyes, Universidad de Chile, Chile Editor: Dr. Luis Campos Medina, Universidad de Chile, Chile

Editores asociados: Dr. Gabriel Felmer, Universidad de Chile, Chile.

\section{Dr. Pablo Navarrete, Universidad de Chile, Chile.}

Dr. Juan Pablo Urrutia, Universidad de Chile, Chile

Coordinadora editorial: Sandra Rivera, Universidad de Chile, Chile.

Asistente editorial: Katia Venegas, Universidad de Chile, Chile.

\section{COMITÉ EDITORIAL:}

Dr. Victor Delgadillo, Universidad Autónoma de la Ciudad de México, México.

Dra. María Mercedes Di Virgilio, CONICET/ IIGG, Universidad de Buenos Aires, Argentina. Dra. Irene Molina, Uppsala Universitet, Suecia.

Dr. Gonzalo Lautaro Ojeda Ledesma, Universidad de Valparaíso, Chile.

Dra. Suzana Pasternak, Universidade de São Paulo, Brasil.

Dr. Javier Ruiz Sánchez, Universidad Politécnica de Madrid, España.

Dra. Elke Schlack Fuhrmann, Pontificia Universidad Católica de Chile, Chile.

Dr. Carlos Alberto Torres Tovar, Universidad Nacional de Colombia, Colombia.

Sitio web: http://www.revistainvi.uchile.cl/

Correo electrónico: revistainvi@uchilefau.cl

Licencia de este artículo: Creative Commons Atribución-Compartirlgual 4.0

Internacional (CC BY-SA 4.0) 